\title{
Routes to self-assembling stable photonic band-gap phases in emulsions of chiral nematics with isotropic fluids
}

\author{
Chien-Yueh Huang \\ Jonathan J. Stott \\ Fairfield University, jstott@fairfield.edu \\ Rolfe G. Petschek
}

Follow this and additional works at: https://digitalcommons.fairfield.edu/physics-facultypubs Copyright 1998 American Physical Society The final publisher PDF has been archived here with permission from the copyright holder.

\section{Peer Reviewed}

\section{Repository Citation}

Huang, Chien-Yueh; Stott, Jonathan J.; and Petschek, Rolfe G., "Routes to self-assembling stable photonic band-gap phases in emulsions of chiral nematics with isotropic fluids" (1998). Physics Faculty

Publications. 116.

https://digitalcommons.fairfield.edu/physics-facultypubs/116

\section{Published Citation}

Huang, Chien-Yueh, Jonathan J. Stott, and Rolfe G. Petschek. "Routes to self-assembling stable photonic band-gap phases in emulsions of chiral nematics with isotropic fluids." Physical review letters 80, no. 25 (1998): 5603.

This item has been accepted for inclusion in DigitalCommons@Fairfield by an authorized administrator of DigitalCommons@Fairfield. It is brought to you by DigitalCommons@Fairfield with permission from the rightsholder(s) and is protected by copyright and/or related rights. You are free to use this item in any way that is permitted by the copyright and related rights legislation that applies to your use. For other uses, you need to obtain permission from the rights-holder(s) directly, unless additional rights are indicated by a Creative Commons license in the record and/or on the work itself. For more information, please contact digitalcommons@fairfield.edu. 


\title{
Routes to Self-Assembling Stable Photonic Band-Gap Phases in Emulsions of Chiral Nematics With Isotropic Fluids
}

\author{
Chien-Yueh Huang, Jonathan J. Stott, and Rolfe G. Petschek \\ Department of Physics, Case Western Reserve University, Cleveland, Ohio 44106
}

(Received 24 February 1998)

\begin{abstract}
Blue phases are stable phases with crystalline packing of interwoven topological defects in chiral nematic liquid crystals. We argue that chiral nematics with appropriate surfactants are likely to form blue phases for a wide range of parameters. We derive the transition curve for stable emulsified blue phases and find that the required low surface tension is within the accessible range of surfactants. These emulsified blue phases provide possible routes to photonic band-gap materials. [S0031-9007(98)06450-3]

PACS numbers: 68.55.Jk, 61.30.-v, 68.10.-m
\end{abstract}

It is known that there are liquid crystalline phases- the blue phases (BP) - that have crystalline structures with lattice constants comparable to the wavelength of light [1-4]. The striking opalescence of these phases led to the first observation of a liquid crystalline phase just over a century ago [5]. These phases occur over only a very limited range of temperature which is related to the large elastic energy associated with the topological defectsdisclinations - that occur in these phases [6-8]. Three distinct blue phases have been found experimentally [912]. The crystalline structures of BP I and II can be understood as a periodic packing of these disclination cores. If the disclination cores and the liquid crystal are viewed as different subphases each subphase is continuous throughout the system, this is an interpenetrating bicontinuous phase [13-15]. However, blue phases have only small variations in the optical dielectric constant. Therefore [3] it is not expected that known blue phases should have photonic band gap.

Stable emulsions in which two incompatible fluids, e.g., oil and water, are mixed using a surfactant have also been known for a long time. These have smaller characteristic length scales and often they are not ordered: the mixture is maintained by entropy and fluctuations in the structure [16-18]. If both phases are isotropic, it is difficult to see how there can be order on scales as long as the wavelength of light as the typical interaction lengths are of the order of a molecular size-around $2 \mathrm{~nm}$ for low molecular weight surfactants and somewhat larger for polymers. An appropriate choice of phases, or by polymerizing one phase and replacing the other phase using supercritical extraction should create very different optical dielectric constants in different regions of the system.

We argue below that if a surfactant can be found that has sufficiently low (but previously realized) surface tension and in addition certain other properties, then an emulsion of a chiral liquid crystalline phase and an isotropic fluid will form with a lattice constant comparable to the wavelength of light and which can be controlled by changing the chirality of the liquid crystal. This length scale is con- trolled not by specific long-range interactions, but rather by the chirality and the effective interactions mediated by the boundary conditions and the slowly changing director of the liquid crystal. Polymerizing the liquid crystalline phase and other appropriate post-processing will then plausibly result in a photonic band gap material - a material in which light with a range of frequencies cannot propagate in any direction [19]. The formation of such an emulsion and photonic band gap materials are of considerable scientific and applied interest.

Blue phases are known to appear in chiral systems with nematic order. The nematic liquid crystals of interest to us are those in which the long axes of rodlike molecules all align locally parallel to a specific direction, "the director." It is known that this director, in the presence of chirality, rotates, leading to cholesteric phases. For sufficiently large chirality, sufficiently close to the isotropicnematic phase transition temperature, this tendency to twist results in blue phases in which the director twists more or less rapidly and in different directions in different parts of the sample [3]. BP have been treated theoretically within a Landau-Ginzburg-like free-energy approximation [20,21]. More appropriately for our purposes, they have been treated as phases in which disclinations form spontaneously [6-8]. Disclinations are topological defects; the nematic director rotates by $180^{\circ}$ along any path encircling a disclination line. To reduce the energy, the molecular alignment tends to zero at the disclination cores. This results in a negative free energy contribution from the bend-twist term in the elastic energy. Meiboom et al. [6] pointed out the importance of this term to blue phases. The elastic energy employed by these authors consists of two terms. The first term is associated with the elastic energy for the nematic director $\mathbf{n}$ :

$$
\begin{gathered}
F_{1}=\frac{1}{2} \int d V\left[K_{s}(\nabla \cdot \mathbf{n})^{2}+K_{t}\left(\mathbf{n} \cdot \nabla \times \mathbf{n}+q_{o}\right)^{2}\right. \\
\left.+K_{b}(\mathbf{n} \times \nabla \times \mathbf{n})^{2}\right],
\end{gathered}
$$

where $K_{s}, K_{t}$, and $K_{b}$ are the positive splay, twist, and bend elastic constants. The chiral pitch of the liquid 
crystal $P=2 \pi / q_{o}$ can be controlled around visible wavelengths without difficulty. The second term is associated with the bend-twist energy:

$$
F_{2}=\frac{1}{2} \int d V\left[\left(K_{t}+K_{24}\right) \nabla \cdot[(\mathbf{n} \cdot \nabla) \mathbf{n}-\mathbf{n}(\nabla \cdot \mathbf{n})]\right],
$$

where $K_{24}$ is the bend-twist elastic constant. When $F_{2}$ is integrated over the disclination core, the surface term does not vanish and instead contributes $-\pi k$ which stabilizes the defects. This assumes the one constant approximation i.e., $k=K_{24}=K_{b}=K_{t}=K_{s}$. Thus the resultant free energy per unit length of the disclination for the blue phase is written as

$$
\mathcal{F}_{\mathrm{M}}=a \Delta T r^{2}+2 \pi r \sigma+\frac{\pi k}{4} \log \frac{R}{r}-\pi k,
$$

where the first term is the core-isotropy energy penalty comprising the latent-heat related coefficient $a$ and the degree of subcooling from the isotropic-cholesteric transition temperature $\Delta T$, the second term is the interfacial energy between the isotropic and the nematic liquid crystals, and the third is the elastic energy associated with the disclination, which has an inner radius $r$ and an outer radius $R$, taken to be the lattice constant or the pitch of the chiral nematic. Minimizing the free energy with respect to $r$, Meiboom et al. concluded the temperature range for blue phases is at most a few degrees.

This free energy changes when an emulsion is considered. The region near the core of a disclination has relatively high free energy and is mostly likely to be replaced by the emulsified isotropic fluid. The surfactant-mediated surface energy must be modeled differently. We will assume planar anchoring, i.e., the nematic director at the surface is always in the plane of the surface. For the interfacial energy we adopt the phenomenological membrane model of Helfrich [22]. We use a Monge representation [17] for the surface function of the membrane in a cylindrical coordinate as $u=\rho-h(\theta, z)$ and the unit normal vector of the membrane is $\nabla u=\hat{\boldsymbol{\rho}}-\frac{1}{\rho} h_{\theta} \hat{\boldsymbol{\theta}}-h_{z} \hat{\mathbf{z}}$ where the magnitude of $\nabla u$ is approximated as unity due to the nearly flat presumption. In Helfrich's expression for the free energy, the most essential contributions to the free energy, to the lowest order, are the surface tension, the surface rigidity, and the membrane asymmetry which is known as the spontaneous curvature. The energy has to be modified to comply with the coupling between the director tensor from the liquid-crystal continuum and the curvature tensor of the membrane. Surfaces between distinct phases tend to curve towards one of the phases [17], e.g., if the surfactant is oriented so that the portion next to the "water" has a larger area than that next to the "oil" then the surface will tend to curve towards the oil. If one of these phases has orientational order it is necessary to take this into account in calculating the tendency to curve. Symmetry arguments show that the interfacial energy is given by

$$
\begin{gathered}
F_{s}=\int d S\left[\sigma+\lambda_{\perp}\left(\nabla^{2} u-\mathbf{n n}: \nabla \nabla u\right)+\lambda_{\|}(\mathbf{n n}: \nabla \nabla u)\right. \\
\left.+\kappa\left(\nabla^{2} u\right)^{2}\right]+\ldots
\end{gathered}
$$

Here $\sigma$ is the effective surface tension; : indicates a double dot product, and $\kappa$ is a bending modulus of the membrane. Several such bending moduli exist, including, e.g., the modulus corresponding to Gaussian curvature $[16,23]$. The distinction between them, like a more general derivation of the membrane free energy, would be important only in a more precise treatment. It is also noted that the last term of the above integration is scale invariant. The tendency of the surface to curve is described by two constants, $\lambda_{\perp}$ and $\lambda_{\|}$: negative $\lambda_{\perp}$ means the surface lowers its free energy by curving towards the isotropic fluid perpendicular to the director; negative $\lambda_{\|}$means the surface lowers its free energy by such curving parallel to the director. To illustrate a possible mechanism for generating differences between the $\lambda$ 's we consider a surfactant consisting of a rigid block and semiflexible tails that dissolve in and align with the nematic liquid crystal. Attached to the rigid block are one or more side chains roughly perpendicular to the block that dissolve in the isotropic fluid phase. There will then be more oil in any surfactant molecule in directions parallel to than perpendicular to the director. Thus the free energy will be lower if the surface curves away from the oil parallel to the director. Consequently, this surfactant membrane is likely to favor a configuration with negative $\lambda_{\|}-\lambda_{\perp}$. The total tendency to curve is controlled (roughly) by the ratio of the effective areas of the parts of the surfactant that dissolve in the oil and water and so can be controlled independently. There are only heuristic guidelines in manipulating physical properties of surfactant membranes even in isotropic fluids. Our example is only one possible scenario. Further exploration of this topic will be required. Note (as seen below) we believe that large negative $\lambda_{\|}$is required for blue phase emulsions. It is convenient to include the surface-effective elastic constants in the surface free energy. Integration by parts confirms that $K_{24}+K_{t}$ can be included as a negative contribution to $\lambda_{\|}$.

First consider structures that vary in three dimensions, e.g., bicontinuous blue phases or phases containing spheres, characterized by a single length-scale $a$ (the lattice constant). The resultant $a$-dependent free energy per unit volume of the liquid crystal from Eqs. (1), (2), and (4) has the form:

$$
\begin{aligned}
\frac{\mathcal{F}}{\phi q_{o}^{2} K_{t}}= & \frac{1}{\hat{a}}\left(\hat{\sigma} f_{\sigma}-f_{P}\right)+\frac{1}{\hat{a}^{2}}\left(\hat{\lambda}_{\perp} f_{\perp}+\hat{\lambda}_{\|} f_{\|}\right. \\
& \left.+\tilde{K}_{s} f_{s}+\tilde{K}_{b} f_{b}+f_{t}\right)+\frac{1}{\hat{a}^{3}}\left(\hat{\kappa} f_{\kappa}\right),
\end{aligned}
$$

where $\phi$ is the volume fraction of the liquid crystal, and the rest of the dimensionless (tilded) coefficients and variables are $\tilde{a}=a q_{o}, \tilde{\sigma}=\sigma / K_{t} q_{o}, \tilde{\lambda}_{\perp}=\lambda_{\perp} / K_{t}, \tilde{\lambda}_{\|}=$ $\lambda_{\|} / K_{t}, \tilde{K}_{s}=K_{s} / K_{t}, \tilde{K}_{b}=K_{b} / K_{t}$, and $\tilde{\kappa}=\kappa q_{o} / K_{t}$. Here $f$ 's are dimensionless integrals over the structure, and for favorable structures we expect $|f| \lesssim 1$. Note that 
$f_{\sigma}, f_{s}, f_{b}, f_{t}$, and $f_{\kappa}$, as integrals of positive functions, are all positive semidefinite. The chiral term $f_{P}$ can be always made positive by choosing the correct chirality. The signs of both $\lambda$ 's and $f_{\perp}$ and $f_{\|}$can be either positive or negative. The elastic constants of liquid crystals are about $k_{B} T / \ell$ where $\ell$ is about the molecular dimension. Therefore, the magnitudes of the $\tilde{K}$ 's are about unity. The value of $\kappa$ will vary within a range from about $100 k_{B} T$ for a rigid lipid membrane to about $1 k_{B} T$ with modification by additives such as cosurfactants $[16,23]$, in which case $\tilde{\kappa}$ is the order of $\ell q_{o}$-less than 0.01 . The value of the $\lambda$ 's will depend on the molecular conformations and interactions among the amphiphiles, and we expect that $\left|\tilde{\lambda}_{\perp}\right|$ is typically less than unity, and $\tilde{\lambda}_{\|}$(including the effect of $K_{24}$ ) is not too far from -1 . Note that the magnitude of $K_{t} q_{o}$ is about $10^{-2}$ dyne $/ \mathrm{cm}$ which is likely an upper bound for the surface tension if blue phases are to form. Emulsification failure occurs when the free energy is less negative than that of the separated phases or $-\phi K_{t} q_{o}^{2}$. As this is the absolute minimum for the elastic energy, we conclude that the contributions from the surface coupling terms (associated with $\lambda$ 's) must be negative enough to sustain a stable blue-phase-like microemulsion. However, these contributions cannot be too large; if the $\tilde{a}^{-2}$ term is negative it will drive the system to a structure with a small characteristic length, $a \sim \ell$-independent of chirality. Therefore $\mathcal{F} / \phi q_{o}^{2} K_{t}$ must be less than but $\mathcal{O}(1)$.

Structures that vary in one dimension, e.g., lamellae, are easily seen to be unfavorable. Structures that vary in two dimensions, e.g., dispersed prisms with length scale $r$ containing a noncontinuous phase in a continuous phase, can be analyzed as in Eq. (5) above. Suppose that the liquid crystal director can be taken to be constant and the prism axis is parallel to it. This implies that the length scale of the prism is small compared to the pitch. It is thus clear that the "best" prism is a cylinder. A water containing cylinder has free energy $\mathcal{F}=2 \pi(\sigma r+$ $\left.\lambda_{\perp}+\kappa / r\right)$ and a liquid crystal containing cylinder has $\mathcal{F}=2 \pi\left(\sigma r-\lambda_{\perp}+\kappa / r\right)$ where $r$ is now the radius of the cylinder. The best value for $r$ is $\sqrt{\kappa / \sigma}$ and the lowest free energy per length is $\mathcal{F}=2 \pi(2 \sqrt{\sigma \kappa}-$ $\left.\left|\lambda_{\perp}\right|\right)$. Therefore if $r=\sqrt{\kappa / \sigma} \leq P$ and $\left|\lambda_{\perp}\right|>2 \sqrt{\kappa \sigma}$ a blue phase will not form, rather the emulsion will consist of small radius tubes within a majority phase. If $r \geq P$, then our assumption that the liquid crystal director is constant is invalid; effectively two-dimensional water prisms are impossible without suppressing the twist; effectively two-dimensional twisted liquid-crystal containing double-twist cylinders are possible. In any case, if $|\lambda| \lesssim k$ and $r \geq P$ the energy associated with an effectively two-dimensional emulsion is of the same order, $\lambda / P$ per distance, as that of a blue-phase emulsion ( $k / P^{2}$ per volume). In consequence, if $r \geq P$ we expect that, at least for approximately equal volume fractions of nematic and isotropic fluid, a three-dimensional emulsion is favored over a two-dimensional one.
Bicontinuous emulsions can be formed with appropriate selections of surfactants, cosurfactants, and salinity, and by lowering the surface tension and bending moduli and adjusting the tendency to curve, e.g., $\lambda$ 's. For microemulsions of isotropic fluids the effect of membrane fluctuation is important [16,24-26]; however, such an effect is suppressed when the surface undulations are coupling with the liquid crystals. Thus, our system is enthalpically controlled and the characteristic length is controlled by the pitch of the chiral nematics.

Detailed calculations for various possibilities regarding the geometry of the emulsion and its crystalline packing are difficult. Here we estimate the range of stable emulsified blue phases by performing a calculation similar to that of Meiboom et al. with a cylindrical disclination core containing isotropic fluid. We will assume that the director around a cylindrical disclination core of radius $r$ has the form $\mathbf{n}=\cos \left(\frac{3 \varphi}{2}\right) \hat{\boldsymbol{\varphi}}-\sin \left(\frac{3 \varphi}{2}\right)[-\sin \theta(\rho) \hat{\boldsymbol{\rho}}+$ $\cos \theta(\rho) \hat{\mathbf{z}}]$ where $\hat{\mathbf{z}}$ is along the axis of the core, $\hat{\boldsymbol{\rho}}$ is along the radial direction, and $\theta(\rho)$ is a variational function. This is consistent with planar anchoring at the surface of the disclination $(\theta=0)$ and with the structure usually ascribed to disclinations in blue phases far from the core [13] $(\theta=\pi / 2)$. In the one constant approximation and making the assumptions of Ref. [6], the free energy difference between the emulsified blue phase and the cholesteric phase with separated isotropic fluid, per unit length of this disclination, is

$$
\begin{aligned}
\mathcal{F}= & 2 \pi\left[r \sigma+\frac{1}{2}\left(\lambda_{\perp}+\lambda_{\|}\right)+0.381 k\right] \\
& +\frac{1}{4} \pi k \log \left(\frac{R}{r}\right)+\frac{\kappa}{r},
\end{aligned}
$$

and must be less than zero for the blue phase to be stable. Comparing Eq. (6) with Eq. (3), note that the explicit temperature dependence disappears, replaced by a contribution from the surface coupling. The temperature dependence in Eq. (6) is much weaker so that the temperature range for stable blue phases is expected to be much larger. The optimal radius of the isotropic core is

$$
r^{*}=\frac{k}{16 \sigma}\left[1+\left(1+\frac{128 \kappa \sigma}{\pi k^{2}}\right)^{1 / 2}\right] .
$$

Introducing $r^{*}$ back to Eq. (6) and setting $R=2 \pi / q_{o}$ and $\mathcal{F}=0$, we obtain the transition curve

$$
\begin{aligned}
\left(\frac{r^{*} q_{o}}{2 \pi}\right)=\exp [ & 3.048+\frac{4}{k}\left(\lambda_{\|}+\lambda_{\perp}\right) \\
& \left.+\left(1+\frac{128 \kappa \sigma}{\pi k^{2}}\right)^{1 / 2}\right] .
\end{aligned}
$$

Below the transition curve, there is a window for the volume fraction $\phi$ within which the emulsified blue phase is stable. Note that the sum of $\lambda$ 's (the effect of the spontaneous curvatures) is crucial not only in balancing the free energy and deciding the stable phases but also in defining the working regime for experimental 
accessibility. If $\kappa \sigma / k^{2}$ is very small, the transition curve can be expressed

$$
\sigma \leq \sigma^{*} \doteq 7 \times 10^{-4} k q_{o} \exp \left[-4\left(\lambda_{\|}+\lambda_{\perp}\right) / k\right] \text {. }
$$

As $k q_{0} \sim 10^{-2}$ dyne $/ \mathrm{cm}$ and $\sigma$ can be made of order $10^{-2}-10^{-3}$ dyne $/ \mathrm{cm}$ this calculation suggests that $\lambda_{\|}$ must be of order $-1.3 k$ to $-1.9 k$ for a blue phase to form. As mentioned before, the $K_{24}$ term is ascribed to the surface coupling and will contribute of order $-k$ to the $\lambda$ 's. It seems reasonable that a similar contribution from the surface can be achieved with appropriate surfactants and additives.

We believe that blue-phase-like structures are also possible if $\lambda_{\|}$is positive. In this case viewing the blue phase as being made of nematic double twist cylinders is more productive. It is easy to see that the free energy of such cylinders is lowered by positive $\lambda_{\|}$.

It is important to note that there is considerable frustration in any bicontinuous emulsion of a nematic and an isotropic fluid. In particular, it is topologically impossible for the director to have the direction that the curvature would prefer everywhere, or for there to be no surface defects "boojums" in the director. For lyotropic liquid crystals, there is a theoretically predicted twist grain boundary (TGB) phase driven by chiral membranes [27]. In a complete investigation of the phase diagram, one should consider all possible geometries of the emulsion [28], topological defects [8,29-31] (e.g., TGB), entropic effects [26,32], etc.

Our analysis strongly suggests that it is possible to make self-assembling blue-phase-like phases from emulsions of chiral nematics, isotropic liquids, and surfactants. This requires conditions previously achieved in bicontinuous microemulsions of isotropic fluids: low surface tensions, appropriate bending moduli, and a small intrinsic curvature $\left|\lambda_{\perp}\right|$. Above we assumed the director at the surface is in the plane of the surface. We do not believe blue phases can form if this director is along the surface normal. However, if this director makes an angle to the surface, blue-phase emulsions may form; this may actually decrease the frustrations in the plane. There are additional, less stringent requirements on ratios of thermodynamic parameters. We believe that either lowmolecular-weight surfactants or polymer surfactants with relatively low rigidity will satisfy the above requirements. Such phases may be further processed to yield photonic band-gap materials.

This research was supported by a grant from the National Science Foundation Science and Technology Center for Advanced Liquid Crystalline Optical Materials (ALCOM) DMR 89-20147. We are grateful to J. Adin Mann, Jr. for useful discussions.

[1] P.G. de Gennes and J. Prost, The Physics of Liquid Crystals (Oxford University Press, New York, 1993), 2nd ed.
[2] P.E. Cladis, in Theory and Applications of Liquid Crystals, edited by J.L. Ericksen and D. Kinderlehrer (Springer-Verlag, New York, 1987), p. 73.

[3] R. M. Hornreich and S. Shtrikman, Mol. Cryst. Liq. Cryst. 165, 183 (1988).

[4] D. C. Wright and N. D. Mermin, Rev. Mod. Phys. 61, 385 (1989).

[5] F. Reinitzer, Monatsh. Chem. 9, 421 (1888).

[6] S. Meiboom, J. P. Sethna, P.W. Anderson, and W.F. Brinkman, Phys. Rev. Lett. 46, 1216 (1981).

[7] J.P. Sethna, in Theory and Applications of Liquid Crystals, edited by J.L. Ericksen and D. Kinderlehrer (Springer-Verlag, New York, 1987), p. 305.

[8] E. Dubois-Violette and B. Pansu, Mol. Cryst. Liq. Cryst. 165, 151 (1988).

[9] P. P. Crooker, Liq. Cryst. 5, 751 (1989).

[10] H. Stegemeyer et al., Liq. Cryst. 1, 3 (1986).

[11] H. Onusseit and H. Stegemeyer, Z. Naturforsch. A 36, 1083 (1981).

[12] M. Marcus, Phys. Rev. A 25, 2272 (1982).

[13] S. Meiboom, M. Sammon, and D.W. Berreman, Phys. Rev. A 28, 3553 (1983).

[14] R. M. Hornreich and S. Shtrikman, Phys. Lett. A 82, 345 (1981).

[15] R. M. Hornreich and S. Shtrikman, Phys. Lett. A 84, 20 (1981).

[16] P. G. de Gennes and C. Taupin, J. Phys. Chem. 86, 2294 (1982).

[17] S. A. Safran, Statistical Thermodynamics of Surfaces, Interfaces, and Membranes (Addison-Wesley, New York, 1994), 1st ed.

[18] G. Gompper and M. Schick, in Phase Transitions and Critical Phenomena, edited by C. Domb and J.L. Lebowitz (Academic Press, London, 1994), 1st ed., Vol. 16.

[19] J. D. Joannopoulos, R. D. Meade, and J. N. Winn, Photonic Crystals (Princeton University Press, New Jersey, 1995), 1 st ed.

[20] R. M. Hornreich and S. Shtrikman, J. Phys. (Paris) 41, 335 (1980).

[21] R. M. Hornreich and S. Shtrikman, Phys. Rev. A 24, 635 (1981).

[22] W. Helfrich, Z. Naturforsch. C 28, 693 (1973).

[23] S. Leibler, in Statistical Mechanics of Membranes and Surfaces, edited by T. Piran, D. Nelson, and S. Weinberg (World Scientific, New Jersey, 1989), p. 45.

[24] W. Helfrich, J. Phys. (Paris) 46, 1263 (1985).

[25] L. Peliti and S. Leibler, Phys. Rev. Lett. 54, 1690 (1985).

[26] S. A. Safran, D. Roux, M.E. Cates, and D. Andelman, Phys. Rev. Lett. 57, 491 (1986).

[27] R. D. Kamien and T. C. Lubensky, J. Phys. II (France) 7, 157 (1997).

[28] L. Golubovic and T. C. Lubensky, Phys. Rev. A 41, 4343 (1990).

[29] N. D. Mermin, Rev. Mod. Phys. 51, 591 (1979).

[30] S. R. Renn and T.C. Lubensky, Phys. Rev. A 38, 2123 (1988).

[31] S. R. Renn and T.C. Lubensky, Phys. Rev. A 41, 4392 (1990).

[32] D. Andelman, M.E. Cates, D. Roux, and S. A. Safran, J. Chem. Phys. 87, 7229 (1987). 